\title{
Maladaptive Alterations of Defensive Response Following Developmental Complex Stress in Rats
}

\author{
Junhyung Kim ${ }^{1,2}$, Minkyung Park ${ }^{2,3}$, Chiheon Lee ${ }^{1,2}$, Jung Jin $\mathrm{Ha}^{4}$, June-Seek Choi ${ }^{5}$, Chul Hoon Kim ${ }^{6}$, \\ Jeong-Ho Seok ${ }^{1,2,3}$ \\ ${ }^{1}$ Department of Psychiatry, ${ }^{2}$ Institute of Behavioral Science in Medicine, ${ }^{3}$ Brain Korea 21 PLUS Project for Medical Science, ${ }^{4}$ Department of \\ Psychology, Yonsei University, ${ }^{5}$ Department of Psychology, Korea University, ${ }^{6}$ Department of Pharmacology, Yonsei University College of \\ Medicine, Seoul, Korea
}

\begin{abstract}
Objective: Despite the etiological significance of complex developmental trauma in adult personality disorders and treatment-resistant depression, neurobiological studies have been rare due to the lack of useful animal models. As a first step, we devised an animal model to investigate the effects of multiple trauma-like stress during different developmental periods.

Methods: Twenty-one male Sprague-Dawley rats were classified into 3 groups based on the stress protocol: fear conditioning control (FCC, $n=6)$, complex stress (ComS, $n=9)$, and control $(n=6)$. While the ComS experienced three types of stress (maternal separation, juvenile isolation, electric foot shock), the FCC only experienced an electric foot shock stress and the control never experienced any. We compared fear responses at postnatal day (PND) 29 and PND 56 through freezing time per episode (FTpE), total freezing time (TFT), total freezing episodes (TFE), and ultrasonic vocalization (USV).

Results: ComS showed the longest FTpE in the conditioned fear response test. ComS and FCC exhibited the longer TFT and these two groups only displayed USV. ComS show difference TFE between PND 29 and PND 56.

Conclusion: The results of this investigation show that complex stress may affect not quantity of fear response but characteristics of fear response. Longer FTpE may be associated with tonic immobility which could be considered as a failed self-protective reaction and might be analogous to a sign of inappropriate coping strategy and self-dysregulation in complex trauma patients.
\end{abstract}

KEY WORDS: Trauma; Animal model; Anxiety; Freezing reaction, cataleptic; Vocalization, animal.

\section{INTRODUCTION}

Human and animal studies have implicated stressful early-life experiences as a key risk factor for the develop-

Received: February 4, 2020 / Revised: March 30, 2020

Accepted: April 10, 2020

Address for correspondence: Jeong-Ho Seok

Department of Psychiatry, Gangnam Severance Hospital, Yonsei

University College of Medicine, 63gil 20, Eonju-ro, Gangnam-gu, Seoul 06227, Korea

E-mail: JOHNSTEIN@yuhs.ac

ORCID: https://orcid.org/0000-0002-9402-7591

*A part of this study has been presented as a poster in the 2018

Annual Spring Meeting of the Korean Neuropsychiatric

Association which had been held in Seoul between the 19th and 20th of April in 2018, and 2018 Annual Meeting of the American Psychiatric Association which had been held in New York between 5th and 9th of May in 2018. This manuscript has not been published elsewhere in part or in entirety and is not under consideration by another journal. ment of psychiatric disorders [1-4]. The term complex trauma refers to multiple, chronic, repetitive experiences of traumatic events, most often in interpersonal nature and early life (e.g., sexual or physical abuse, neglect, war, community violence) $[5,6]$. Complex trauma affects victims throughout their entire life, and children exposed to complex trauma may show increased vulnerability to additional trauma and cumulative impairment $[5,7]$. Complex trauma also exhibits a strong association with the development of chronic treatment-resistant depression and borderline personality disorder in adulthood [8]. Despite its adverse effects, complex trauma in children is prevalent in the community [9-11].

For the development of effective intervention programs, studies have indicated that a better understanding and assessment of the mental health of patients with complex

(c) This is an Open-Access article distributed under the terms of the Creative Commons Attribution Non-Commercial License (http://creativecommons.org/licenses/by-nc/4.0) which permits unrestricted non-commercial use, distribution, and reproduction in any medium, provided the original work is properly cited. 
trauma are needed [9]. Patients with complex trauma are known to suffer from several psychiatric problems, including post-traumatic stress disorder (PTSD) and other comorbid disorders and functional impairments [5,12]. However, previous studies have only focused on specific, limited aspects of complex trauma, such as suicidality and early childhood regulatory and attachment disorders, and PTSD [13-15]. More recent studies have demonstrated that complex trauma affects the chronic self-regulation problems associated with maladaptive coping strategies and the capacity of self for altering one's behavior $[5,16,17]$. According to the description of complex PTSD in the International Classification of Disease, 11th version (ICD-11), complex trauma encompasses commonly prolonged or repetitive events from which escape is difficult or impossible (e.g., torture, slavery, genocide campaigns, prolonged domestic violence, repeated childhood sexual or physical abuse). Moreover, it is also characterized by severe and persistent 1) problems in affect regulation; 2) beliefs about oneself as diminished, defeated or worthless, accompanied by feelings of shame, guilt, or failure related to the traumatic event; and 3) difficulties in sustaining relationships and in feeling close to others [18]. Therefore, in order to understand the various aspects of complex trauma, it is necessary to understand self dysregulation in the patients of complex trauma.

Researchers have studied the immediate and long-term results of complex trauma, both biologically and behaviorally. While the neural basis of these abnormalities has not been fully elucidated, previous studies have suggested an association between childhood trauma and structural and functional abnormalities of brain regions mediating emotion, such as the hippocampus, amygdala, and prefrontal cortex [19-22]. However, there are limitations in population studies, because inducing trauma to humans has ethical issues and because it is difficult to carry out well-validated behavioral and psychophysiological studies in human populations. Therefore, previous studies of trauma surveying the neurobiological and genetic basis of anxiety and depression have used several animal models $[23,24]$.

However, previous studies using animal models of complex trauma have some limitations. First, most animal models were based on both a single period and a single type of stress. Considering the life cycle, complex trauma in human life during perinatal, infancy, and juvenile peri- od may correspond to that of rats in the prenatal, postnatal, and juvenile periods [25]. Maternal separation (MS) is a commonly used method to induce postnatal stress in rats $[26,27]$. Subjecting rats to MS can result in lasting changes in various measures of emotion-related behavior and stress-reactivity [28]. Other studies have focused on the long-term effects of several interventions on the prepubertal period (postnatal day [PND] 21 to PND 30-34) [29]. Juvenile isolation induces a variety of symptoms in rats, including depression-, anxiety-, and psychosis-like behaviors and signs of autonomic, neuroendocrine, and metabolic dysregulation [30-32]. Electric foot shock has also been incorporated as a stressor in various animal models of human disease, including anxiety, PTSD, and depression [33,34]. Second, although there are previous studies that have examined the effect of stress over different developmental stages $[35,36]$, no previous studies have employed different stressors across different developmental periods, which is a hallmark feature in human complex trauma victims. In addition thereto, the results from currently used animal models are not uniform [37], and it also has been argued that each model of rats has a good ecological validity to model human mental illness [38]. Together, it will be helpful to employ multiple stressors across multiple development periods for building the animal model of complex trauma.

Because complex trauma makes patients vulnerable to additional victimization in other risky environments [16], we wanted to investigate how complex trauma maladaptively affects the defensive response in trauma-like environments. Typical behavior measurements in rat animal models (open field test, elevated plus maze and forced swimming test) are usually known to measure general and sustained symptoms, such as anxiety, unconditioned avoidance - approach behavior, and decreased locomotor activity in depression [39-41], rather than directly measuring behaviors related to the trauma-like situation. In contrast, fear conditioning reactions are defensive responses in the environment associated with stress [42], which can reflect vulnerability to further victimization, and a fear conditioning paradigm was developed to show the effects of chasing stress on sensitization to unconditioned stimuli [43]. Thus, by examining the effects of complex stress on the fear conditioning response, it may be possible to explore more directly the maladaptive alterations of complex stress in the defensive re- 
sponse associated with further victimization.

Complex trauma is an important subject in psychiatry. Although an appropriate animal model is needed for the study of complex trauma, no animal model has been developed to demonstrate various traumas over several periods. In this study, we developed an animal model for studying complex trauma by applying different stressors at individual developmental stages. To examine the validity of the developed animal model, we examined anxiety-related behavioral characteristics.

\section{METHODS}

\section{Animals and Housing}

All experiments were conducted with offspring of three pregnant female Sprague-Dawley (SD) rats obtained from Orientbio Inc. (http://www.orient.co.kr/common/main.asp). Pregnant females at the first week of gestation period were individually transferred and given a 2-week habituation period. Ten to fifteen rats were born to each single parent. To exclude the effects of hormonal change, only male littermates were used in this study. The rats were housed in a climate-controlled laboratory environment $\left(22 \pm 1^{\circ} \mathrm{C}\right)$ under a 12 hours light/dark cycle (lights on at 7:0019:00) with ad libitum access to food and water. On PND 2, male littermates from three parents were assigned to three groups, with male littermates from the same parent assigned to the same group: fear conditioning control ( $n=$ $6)$, complex stress $(n=9)$, or control $(n=6)$. The pups were weaned on PND 21 and housed under standard conditions, except the complex stress group (as described below). Stress protocols of each group are depicted in Figure 1A. The fear conditioning control group (FCC) experienced only electric foot shock stress at PND 28, and the control group never experienced any stress throughout the experiment. Both groups (FCC and control) were housed under standard conditions without any interventions during stress protocols. In the complex stress (ComS) group, all pups experienced three types of stress (maternal separation, juvenile isolation, electric foot shock). All animal procedures were performed in accordance with the US National Institutes of Health Guide for Care and Use of Laboratory Animals and approved by the Institutional Animal Care and Use Committee of the Yonsei University Health System (approval no. 20170003).

\section{Separation Stress Protocol (Postnatal and Juvenile)}

For conveying maternal separation (MS) in the neonatal period (PND 0-21), pups of the ComS group were removed from the home cage and placed inside incubators for 3 hours (10:00-13:00) per day during PND 2-14, while pups in the other groups were maintained together with their littermates. The duration and period of separation were based on MS protocols in other studies [44,45]. Incubators were pre-warmed and maintained at $30 \pm 1^{\circ} \mathrm{C}$ using an adjustable heat mat during separation for avoiding hypothermia. For accentuating stress, separated pups were physically separated from one another during maternal separation. After 3 hours of separation, pups were handled individually during transfer and, when returned, placed into the corner opposite of the nest.

Male SD rats in the ComS group were housed either individually in the same cages under standard conditions (as described 2.1) for 1 week from PND 21 (weaning age corresponding to pre-adolescence) to induce stress related to social isolation in the juvenile period (juvenile isolation) [30]. After 1 week of isolation, nine male SD rats were re-housed together under conventional housing conditions, in which three juvenile rats were housed together until PND 42 and each rat had been housed in a single cage from PND 43. The protocol for juvenile isolation was based on other previous studies $[30,46]$. The separation stress protocol is depicted in Figure 1B.

\section{Foot Shock Stress and Fear Conditioning Protocol}

To induce an excessive traumatic experience, rats in the ComS and FCC groups were transferred to the testing room and underwent experiments from 17 o'clock in the prepubertal period (PND 28). The foot shock experiment was performed using a computerized fear-conditioning system (Panlab startle and fear conditioning system; Harvard Apparatus, Holliston, MA, USA). After 3 minutes of habituation, the animals were presented with inescapable tone-foot shock pairings (pure tone: 30 seconds, $55 \mathrm{~dB}$; electric foot shock: $1.0 \mathrm{~mA}, 2$ seconds) three times at 30-seconds intervals. During the last 1 minute after the foot shock phase, no stimulus was presented [47]. Over a total of 7 minutes, locomotor activity and vocalization were recorded by a camera mounted on the rectangular foot shock chamber $(250$ [width] $\times 250$ [depth] $\times 250$ [height] mm). After each individual test session, the apparatus was completely cleaned with $70 \%$ alcohol to elimi- 
A Trauma protocol of each group

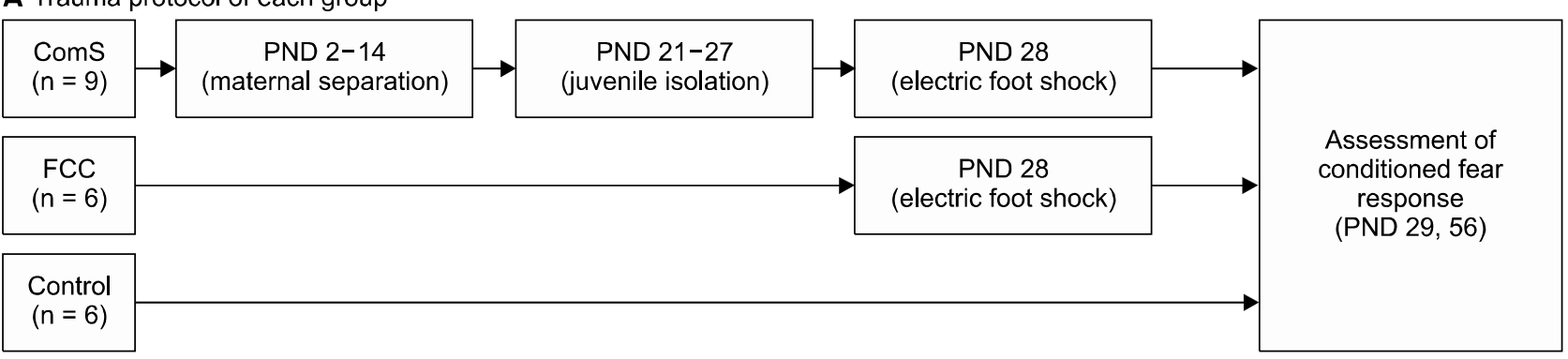

B Separation stress protocol of complex stress group

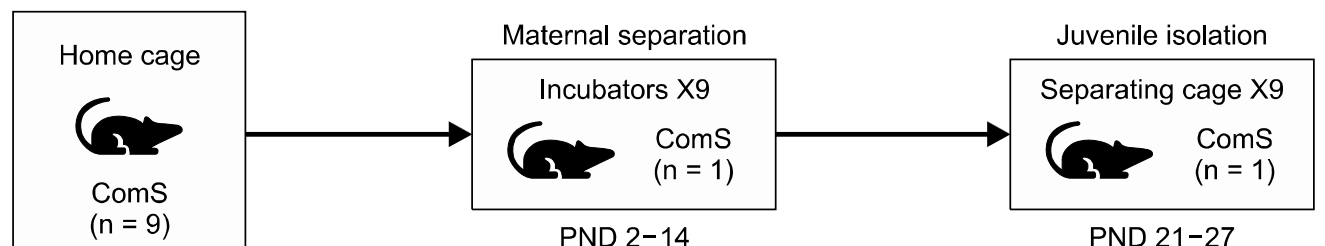

C Foot shock stress and fear conditioning protocol (PND 28)

CS: $55 \mathrm{~dB}$ pure tone

US: $1.0 \mathrm{~mA}$ electric foot shock $(2 \mathrm{sec})$

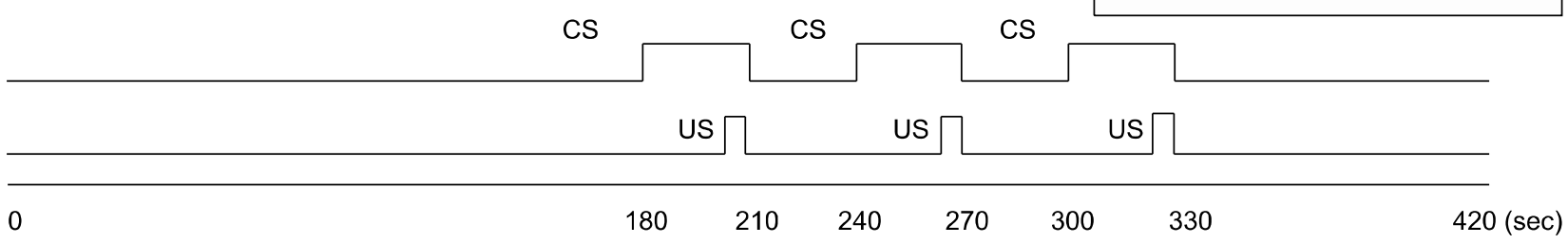

D Assessment protocol of conditioning fear response (PND 29, 56)

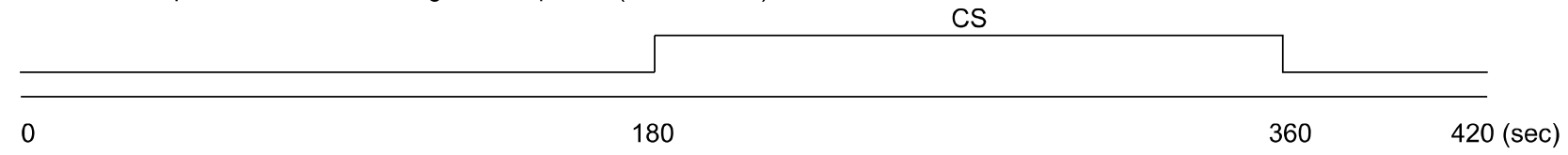

Fig. 1. Schematic representation of the protocol. (A) Overview of the stress protocols of each group. (B) Separation stress protocol of the Complex stress group. (C) Foot shock stress and fear conditioning protocol. (D) Assessment protocol of conditioned fear response.

ComS, complex stress; FCC, fear conditioning control; PND, postnatal day; CS, conditioned stimulus; US, unconditioned stimulus.

nate odor and defecation from the previous tested rats (Fig. 1C).

\section{Assessment Protocol of Conditioned Fear Response}

To examine the acute and lasting conditioned fear response, fear conditioning experiments were conducted at 1 day (PND 29) and 4 weeks (PND 56) after the foot shock stress [47]. All groups (including the control group) were assessed for conditioned fear responses in the same environment to the fear conditioning. This protocol differs from the previous fear conditioning protocol in that it only presents pure tone without paring electric shocks. Animals were presented with 3 minutes of pure tone and 1 minute without any auditory stimulus after a 3-minutes habituation period. After each test session, the apparatus was completely cleaned with $70 \%$ alcohol to eliminate odor and defecation from the previously tested rats (Fig. 1D).

\section{Behavioral Data Collection}

We collected data measured at PND 29 and PND 56 and used the Any-maze ${ }^{\circledR}$ (Stoelting Co., Wood Dale, IL USA) behavioral tracking software to detect a freezing response in the foot shock chamber. We recorded every test session using a camera mounted on the top of the chamber. The video files were transferred to the Any-maze ${ }^{\circledR}$ program, which automatically analyzed total freezing 
time (TFT) and total freezing episode (TFE). Freezing was defined as a total absence of a body or head movement, except that associated with breathing [48]. A single "freezing episode" was defined by continuous freezing behavior over 2 seconds [49]. From TFT and TFE, freezing time per episode (FTpE) was calculated. Additionally, for measuring fear and anxiety related to conditioned fear responses, acoustical analysis of ultrasonic vocalization (USV) was used: we used the Petterson D-230 Bat Detector, which transforms high-frequency sounds (22 kHz) into the audible range [50]. The audio files were opened on Adobe Audition 3.0, and we filtered out any noise but USV. The total time of USV only during the 3-minutes tone in the conditioned fear response test was measured by an experienced user.

\section{Statistical Analysis}

The data are presented as means \pm standard deviations of values following normal distribution and medians and ranges for those following non-normal distribution in Table 1. The data were analyzed using IBM SPSS Win version 23.0 (IBM Co., Armonk, NY, USA). For comparing the freezing behavior measurements of all groups that followed non-normal distribution, Kruskal-Wallis test and post hoc analysis including multiple pairwise comparisons adjusted by the Bonferroni procedure was used. For the USV time extracted from normally distributed variables, repeated measures of analysis of variance (ANOVA) with between subject factors (i.e., stress conditions [FCC, ComS]) and within subject factors (i.e., time [PND 29, 56]) was used for analysis, and the Bonferroni's method and paired $t$ test were applied for post hoc test. Longitudinal comparison of freezing behavior measurements of each group was conducted by paired $t$ test for values following normal distribution and by Wilcoxon signed rank test for values following non-normal distribution. A $p$ value of 0.05 was set as the level of significance.

\section{RESULTS}

\section{Freezing Time per Episode}

FTpE, TFT, TFE, and USV at PND 29 and PND 56 are shown in Table 1. Statistical analysis revealed that FTpE was significantly different among groups at PND 29 ( $p<$ $0.001)$ and PND $56(p<0.001)$. In the post hoc test for $\mathrm{FTpE}$, ComS rats showed the longest time per episode than any of the other groups at PND 29 (FCC: $p=0.015$, control: $p<0.001)$. The ComS and FCC group rats froze longer than the controls at PND 56 (Coms: $p<0.001$, FCC: $p=0.027)$; however, there was no significant difference in FTpE at PND 56 between ComS and FCC ( $p=$ 0.339). There was no significant difference in FTpE between PND 29 and PND 56 in all groups (Coms: $p=$ 0.066, FCC: $p=0.345$, control: $p=0.593$ ) (Fig. 2).

\section{Total Freezing Time}

TFT at both PND 29 and PND 56 were significantly different among groups (PND 29: $p<0.001$, PND 56: $p<$ 0.001). In post hoc test, the ComS and FCC groups displayed longer TFT than controls at PND 29 (Coms: $p<$ 0.001, FCC: $p=0.002$ ) and PND 56 (Coms: $p<0.001$, FCC: $p=0.004)$, although there was no difference in TFT between ComS and FCC rats in either PND $29(p=0.816)$ or PND 56 ( $p=0.339)$. There was no significant differ-

Table 1. Freezing behavior and USV at PND 29 and PND 56

\begin{tabular}{|c|c|c|c|c|}
\hline \multirow{2}{*}{ Variables } & \multirow{2}{*}{ PND } & \multicolumn{3}{|c|}{ Group } \\
\hline & & ComS $(n=9)$ & $\operatorname{FCC~}(n=6)$ & Control $(n=6)$ \\
\hline \multirow[t]{2}{*}{ FTpE } & PND 29 & $59.80(13.80-179.70)$ & $13.72(10.06-24.22)$ & $0(0-7.20)$ \\
\hline & PND 56 & $17.05(6.22-89.65)$ & $9.72(2.98-44.75)$ & $0(0-3.98)$ \\
\hline \multirow[t]{2}{*}{ TFT } & PND 29 & $171.90(41.40-179.70)$ & $118.20(80.50-147.80)$ & $0(0-7.20)$ \\
\hline & PND 56 & $155.00(80.80-179.30)$ & $117.10(17.90-179.00)$ & $0(0-19.90)$ \\
\hline \multirow[t]{2}{*}{ TFE } & PND 29 & $3(1-5)$ & $9.00(5-10)$ & $0(0-3)$ \\
\hline & PND 56 & $9(2-14)$ & $11.00(4-15)$ & $0(0-5)$ \\
\hline \multirow[t]{2}{*}{ USV } & PND 29 & $20.52 \pm 30.44$ & $24.18 \pm 26.43$ & \\
\hline & PND 56 & $74.32 \pm 47.34$ & $101.88 \pm 36.59$ & \\
\hline
\end{tabular}

Values are presented as mean \pm standard deviation or median (interquartile range).

USV, ultrasonic vocalization (sec); PND, postnatal day; ComS, complex stress; FCC, fear conditioning control; FTpE, freezing time per episode (sec); TFT, total freezing time (sec); TFE, total freezing episode. 


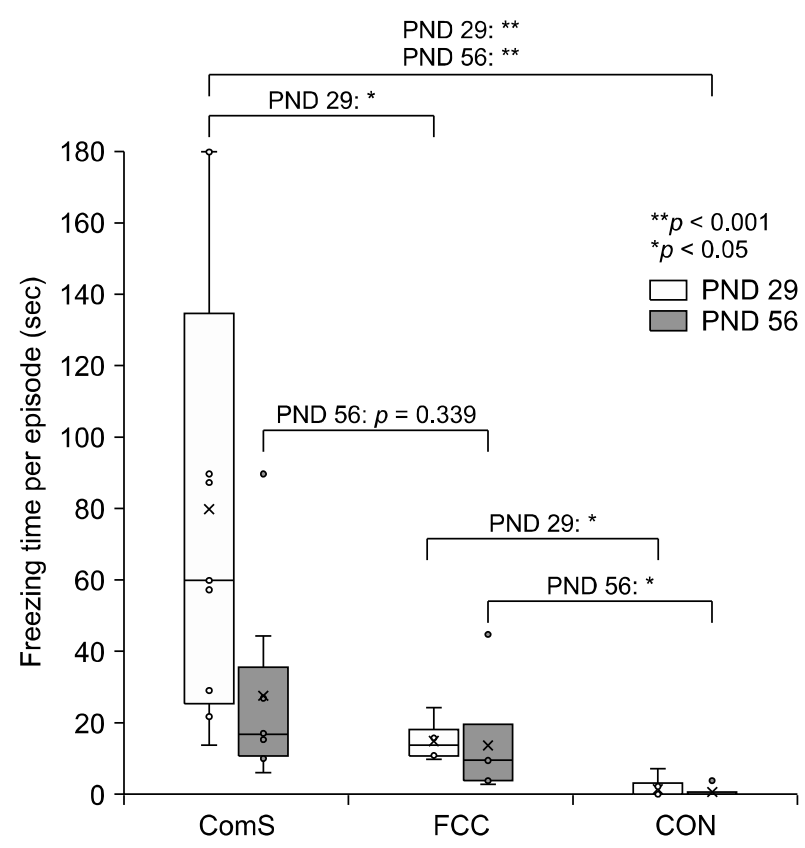

Fig. 2. The effect of each stress condition on freezing behavior in FTpE of each group. For each box, the central mark shows the median, the edges of the box represent the 25th and 75th percentiles, and the whiskers extend to the most extreme data points that are not considered outliers. The ends of the whiskers represent the lowest data value still within 1.5 times the interquartile range (IQR) of the lower quartile and the highest data value still within 1.5 times the IQR of the upper quartile. Kruskal-Wallis test. Exposure to the complex stress condition increased FTpE, compared to a single stress condition or no stress.

FTpE, freezing time per episode; ComS, complex stress; FCC, fear conditioning control; CON, control; PND, postnatal day.

ence in TFT between PND 29 and PND 56 in all groups (Coms: $p=0.678$, FCC: $p=0.753$, control: $p=1.000$ ) (Fig. 3).

\section{Total Freezing Episode}

There were significant differences in TFE at both PND $29(p=0.001)$ and PND $56(p=0.004)$. In the post hoc test, ComS and FCC rats show significantly increased TFE, compared to controls, at PND 29 (ComS: $p=0.018$, FCC: $p=0.002)$ and PND 56 (Coms: $p=0.002$, FCC: $p=$ 0.004). There was a significant difference in TFE between Coms and FCC rats only at PND $29(p=0.001)$, not at PND $56(p=1.000)$. There was a significant difference in TFE between PND 29 and PND 56 only in the ComS group (Coms: $p=0.013$, FCC: $p=0.416$, control: $p=$ 1.000) (Fig. 4).

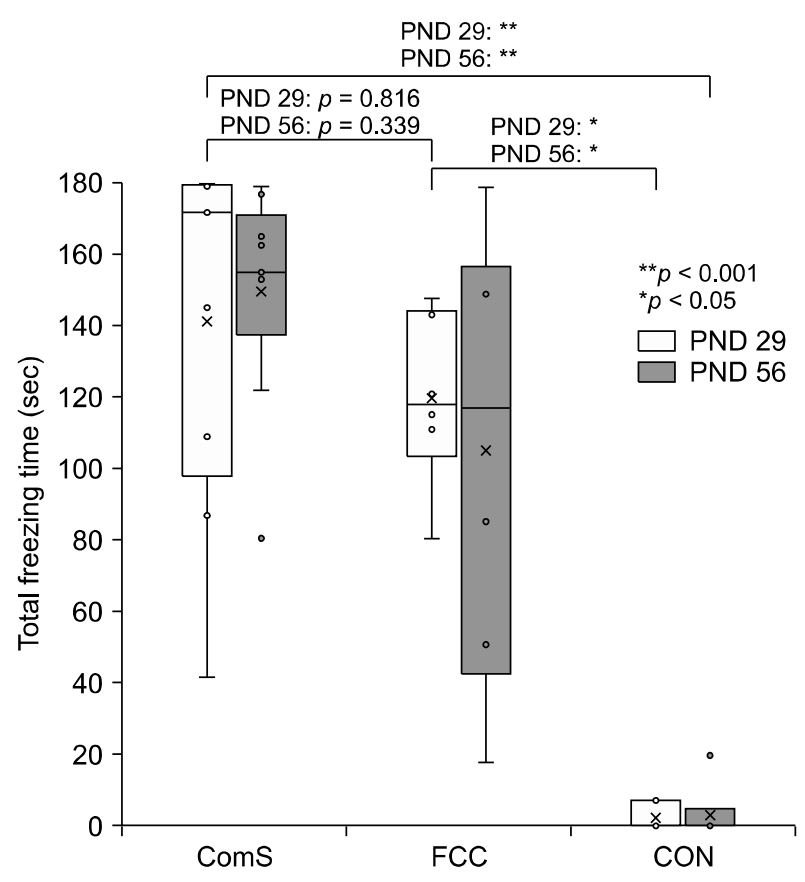

Fig. 3. The effect of each stress condition on freezing behavior in TFT for each group. For each box, the central mark shows the median, the edges of the box represent the 25th and 75th percentiles, and the whiskers extend to the most extreme data points that are not considered outliers. The ends of the whiskers represent the lowest data value still within 1.5 times the interquartile range (IQR) of the lower quartile and the highest data value still within 1.5 times the IQR of the upper quartile. Kruskal - Wallis test. ComS and FCC rats froze significantly more time than controls.

TFT, total freezing time; ComS, complex stress; FCC, fear conditioning control; CON, control; PND, postnatal day.

\section{Ultrasonic Vocalization}

ComS and FCC groups of rats emitted spontaneous USV, while the control group did not. Repeated measure ANOVA showed the main effect of time ( $\mathrm{F} 1=25.842, p<$ 0.001); however, there were no significant interactions between group and time $(\mathrm{F} 1=0.841, p=0.376)$ and no group effects (F4 =1.161, $p=0.301$ ) (Fig. 5).

\section{DISCUSSION}

In this study, we attempted to show for the first time that complex stress exposure may have a differential effect on conditioned fear responses as an anxiety-related behavior in rats. ComS rats showed significantly longer FTpE than other rats. Because freezing episode was defined as freezing over 2 seconds without motion in this study, we considered that a long freezing time per single episode might be associated with tonic immobility $(\mathrm{TI})$, which is a sus- 


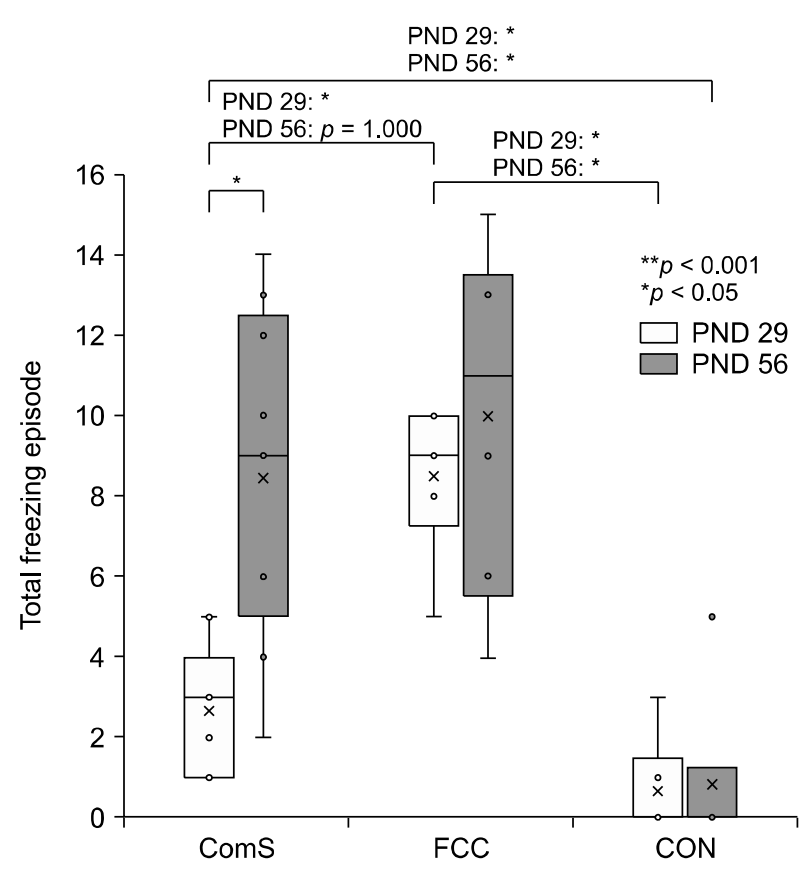

Fig. 4. The median TFE of ComS, FCC, and control groups. For each box, the central mark shows the median, the edges of the box represent the 25th and 75th percentiles, and the whiskers extend to the most extreme data points that are not considered outliers. The ends of the whiskers represent the lowest data value still within 1.5 times the interquartile range (IQR) of the lower quartile and the highest data value still within 1.5 times the IQR of the upper quartile. Kruskal-Wallis test. ComS only increased in TFE at PND 56, compared to PND 29.

TFE, total freezing episode; ComS, complex stress; FCC, fear conditioning control; $\mathrm{CON}$, control; PND, postnatal day.

tained, profound, and reversible physical immobility. TI in humans is also presented as a sustained and largely involuntary pattern of neuromuscular activity (i.e., cataleptic-catatonic) $[51,52]$. If longer FTpE can be considered as an indicator of $\mathrm{TI}$, this result may suggest that complex stress is associated with TI.

Compared to the control group, the Coms and FCC groups exhibited longer TFT, and only these two groups displayed USV. The behavioral profiles in these tests were consistent with previous studies demonstrating increased defensive responses in fear conditioning with tone and foot shock following traumatic stress treatment [47]. In the post hoc test, Coms rats did not show a significant difference in TFT and USV time, compared to FCC rats. One possible explanation for this result is a ceiling effect: TFT was skewed towards the upper bounding time of $180 \mathrm{sec}$ onds (Fig. 1) in both ComS and FCC rats. This result may also be explained by the difference in the effect of complex stress in characteristics of fear response. We can sur-

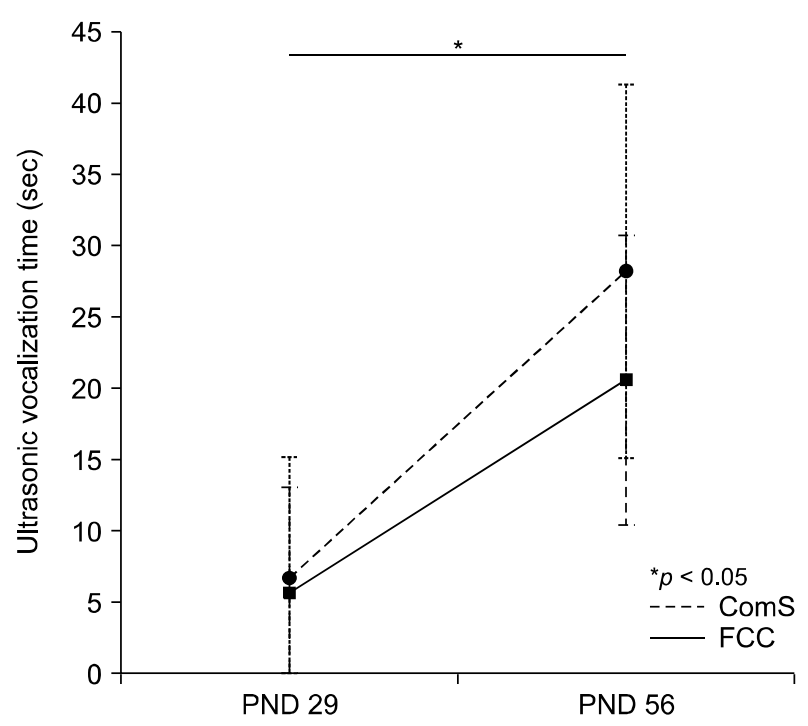

Fig. 5. Ultrasonic vocalization time for ComS and FCC rats at PND 29 and PND 56. Both groups displayed an increased ultrasonic vocalization time at PND 56, compared with PND 29.

ComS, complex stress; FCC, fear conditioning control; PND, postnatal day.

mise that complex stress may not affect the total quantity of a fear response, but may affect the characteristic of fear responses. However, due to limitations of this study's design, their respective contributions cannot be disentangled.

Only ComS rats showed a difference in TFE between that at PND 29 and that at PND 56, and there was no difference in TFE at PND 56 between ComS and FCC rats. This result may imply that freezing behavior in ComS rats at PND 29 was qualitatively different from freezing behaviors in other groups and at other assessment times. Previous fear conditioning research showed that conditioned fear responses lasted more than 4 weeks after electric foot shock $[47,53]$. Therefore, it can be assumed that additional factors other than electric foot shock might affect ComS, showing a different TFE at PND 29. Because both ComS and FCC rats were in a frozen state most of the time, decreased TFE meant sustained, ongoing general physical immobility. Therefore, decreased TFE might be associated with $\mathrm{TI}$, which is defined as ongoing general physical immobility [40]. These results may also be linked to previous work comparing dissociation in a clinical population who were sexually assaulted and $\mathrm{TI}$ in animals [54-56]. Because TI is especially associated with failure of integrating information and reacting appropriately and loss of chance to avoid further damage, TI can lead ComS group accident-proneness [57-59]. 
Additionally, the control group exhibited no USV. Because the control group did not experience any stress protocol, including electric foot shock as a negative control, this result supported the validity of the experiment. Unlike in FTpE of ComS (Fig. 2), USV time did not show a statistically significant difference between ComS and FCC groups, even though the ComS group showed increasing USV over time (Fig. 5). This finding may be interpreted as FTpE and USV reflecting different aspects of fear- and anxiety-related behaviors. The difference in incremental trends in USV and freezing behavior has also been shown in other studies of fear conditioning. In other studies, USV was more prevalent during inter-trial intervals of fear conditioning sessions than during the presence of the conditioned stimulus [55], and the amount of freezing reflected the strength of conditioned stimuli [60]. Suppressed vocal behavior was also described as a characteristic of TI in previous research $[61,62]$. It is likely that FTpE is related to more intense fear-related behavior than that of USV. However, as freezing time per episode was not studied widely as a feature of fear response, the interpretation of this result requires replication in future studies.

We did not directly observe other catatonic features in the freezing state, which is a limitation obscuring our interpretation of FTpE as being strongly associated with $\mathrm{TI}$ (i.e., hypertonicity, catatonic-like motionless posture, unresponsiveness to painful stimulation) $[63,64]$. It is necessary to involve conventional measurements for anxietyrelated behavior (open field test, forced swimming test, and elevated plus maze) to understand the effects of complex trauma more comprehensively and to validate FTpE as a tool for evaluating anxiety in future study. Not investigating behavior in the fear conditioning experiment with rats that experienced only maternal separation and juvenile isolation is a limitation to interpreting the results of the current study. Because this study included only a small number of male rats, the question about possible sex differences in the outcome of complex stress could not be ascertained, and there is a limitation to generalizing interpretations of this study. Given the difference between female and male rats, further studies of the effects of hormonal changes in females are essential. Furthermore, evaluating objective measures in the animal model is also necessary to confirm the validity of the animal model in the current study. Therefore, further study should examine FTpE with fear response, including TI, more directly by in- vestigating other features of fear responses with larger samples and with objective measures, including cortisol and epigenetic changes in experimental animals

This is the first study to investigate the possibility of differential fear responses in a complex stress animal model. We noted increased FTpE at PND 29, compared to PND 56 , in only ComS rats, not in the others. We suggest that longer FTpE may be associated with $\mathrm{TI}$, which may be considered as a sign of inappropriate coping strategies in complex trauma patients. In addition, because inappropriate coping strategies, such as dissociation, make patients vulnerable to additional victimization, we also suggest that increased FTpE and decreased TFE at PND 29 may hold important meaning in research of the effect of complex stress in animal models.

\section{Acknowledgments}

This research was supported by the Basic Science Research Program through the National Research Foundation of Korea (NRF) funded by the Ministry of Education (2017R1D1A1B03029870) and a faculty research grant from Yonsei University College of Medicine (6-2015-0059).

\section{- Conflicts of Interest}

No potential conflict of interest relevant to this article was reported.

\section{Author Contributions}

Conceptualization: Junhyung Kim, June-Seek Choi, Chul Hoon Kim, and Jeong-Ho Seok. Data acquisition: Minkyung Park, Chiheon Lee, Jung Jin $\mathrm{Ha}$, and Jeong-Ho Seok. Formal analysis: Junhyung Kim. Funding: Jeong-Ho Seok. Writing - original draft: Junhyung Kim, Minkyung Park, and Jeong-Ho Seok. Writing-review \& editing: Junhyung Kim and Jeong-Ho Seok.

\section{ORCID}

Junhyung Kim Minkyung Park https://orcid.org/0000-0001-7123-9535 Chiheon Lee https://orcid.org/0000-0002-7896-1640 Jung Jin $\mathrm{Ha}$ June-Seek Choi https://orcid.org/0000-0001-6240-9504 https://orcid.org/0000-0002-7902-5512 Chul Hoon Kim https://orcid.org/0000-0002-4394-2140 Jeong-Ho Seok https://orcid.org/0000-0002-7360-429X https://orcid.org/0000-0002-9402-7591 


\section{REFERENCES}

1. LeMoult J, Humphreys KL, Tracy A, Hoffmeister JA, Ip E, Gotlib IH. Meta-analysis: exposure to early life stress and risk for depression in childhood and adolescence. J Am Acad Child Adolesc Psychiatry 2019. doi: 10.1016/j.jaac.2019. 10.011. [Epub ahead of print]

2. Martins-Monteverde CMS, Baes CVW, Reisdorfer E, Padovan T, Tofoli SMC, Juruena MF. Relationship between depression and subtypes of early life stress in adult psychiatric patients. Front Psychiatry 2019;10:19.

3. Dallé E, Mabandla MV. Early life stress, depression and Parkinson's disease: a new approach. Mol Brain 2018;11:18.

4. Young EA, Abelson JL, Curtis GC, Nesse RM. Childhood adversity and vulnerability to mood and anxiety disorders. Depress Anxiety 1997;5:66-72.

5. Cook A, Spinazzola J, Ford J, Lanktree C, Blaustein M, Cloitre $\mathrm{M}$, et al. Complex trauma in children and adolescents. Psychiatr Ann 2005;35:390-398.

6. Sedlak AJ, Hantman I, Schultz D. The third national incidence study of child abuse and neglect (NIS-3): public use file manual. Washington:US Department of Health and Human Services; 1997.

7. van der Kolk BA. Developmental trauma disorder: toward a rational diagnosis for children with complex trauma histories. Psychiatr Ann 2005;35:401-408.

8. Jumper SA. A meta-analysis of the relationship of child sexual abuse to adult psychological adjustment. Child Abuse Negl 1995; 19:715-728.

9. Spinazzola J, Ford JD, Zucker M, van der Kolk BA, Silva S, Smith SF, et al. Survey evaluates: complex trauma exposure, outcome, and intervention among children and adolescents. Psychiatr Ann 2005;35:433-439.

10. Do J, Woo J. From gut to brain: alteration in inflammation markers in the brain of dextran sodium sulfate-induced colitis model mice. Clin Psychopharmacol Neurosci 2018;16:422-433.

11. Muneer A, Minhas FA. Telomere biology in mood disorders: an updated, comprehensive review of the literature. Clin Psychopharmacol Neurosci 2019;17:343-363.

12. Gekker M, Coutinho ESF, Berger W, Luz MPD, Araújo AXG, Pagotto LFADC, et al. Early scars are forever: childhood abuse in patients with adult-onset PTSD is associated with increased prevalence and severity of psychiatric comorbidity. Psychiatry Res 2018;267:1-6.

13. Scheering MS, Zeanah $\mathrm{CH}$. A relational perspective on PTSD in early childhood. J Trauma Stress 2001;14:799-815.

14. Kinniburgh KJ, Blaustein M, Spinazzola J, van der Kolk BA. Attachment, self-regulation, and competency: a comprehensive intervention framework for children with complex trauma. Psychiatr Ann 2005;35:424-430.

15. Campbell A, Balaratnasingam S, McHugh C, Janca A, Chapman M. Alarming increase of suicide in a remote Indigenous Australian population: an audit of data from 2005 to 2014.
World Psychiatry 2016;15:296-297.

16. Baumeister RF, Vohs KD. Self-regulation, ego depletion, and motivation. Soc Personal Psychol Compass 2007;1:115-128.

17. Ryan K, Lane SJ, Powers D. A multidisciplinary model for treating complex trauma in early childhood. Int I Play Ther 2017;26:111-123.

18. WHO. ICD-11 for mortality and morbidity statistics [Internet]. Gavana: WHO; 2018 [cited at 2019 Mar 2]. Available from: https://icd.who.int/browse11/l-m/en\#/http\%3a\%2f\%2fid.wh o.int\%2ficd\%2fentity\%2f435227771.

19. Herzog JI, Niedtfeld I, Rausch S, Thome J, Mueller-Engelmann $\mathrm{M}$, Steil R, et al. Increased recruitment of cognitive control in the presence of traumatic stimuli in complex PTSD. Eur Arch Psychiatry Clin Neurosci 2019;269:147-159.

20. Boccia M, D’Amico S, Bianchini F, Marano A, Giannini AM, Piccardi L. Different neural modifications underpin PTSD after different traumatic events: an fMRI meta-analytic study. Brain Imaging Behav 2016;10:226-237.

21. Schlumpf YR, Nijenhuis ERS, Klein C, Jäncke L, Bachmann S. Functional reorganization of neural networks involved in emotion regulation following trauma therapy for complex trauma disorders. Neuroimage Clin 2019;23:101807.

22. Teicher MH, Andersen SL, Polcari A, Anderson CM, Navalta $\mathrm{CP}$, Kim DM. The neurobiological consequences of early stress and childhood maltreatment. Neurosci Biobehav Rev 2003;27:33-44.

23. Schmidt MV, Wang XD, Meijer OC. Early life stress paradigms in rodents: potential animal models of depression? Psychopharmacology (Berl) 2011;214:131-140.

24. Goodwill HL, Manzano-Nieves G, Gallo M, Lee HI, Oyerinde $\mathrm{E}$, Serre T, et al. Early life stress leads to sex differences in development of depressive-like outcomes in a mouse model. Neuropsychopharmacology 2019;44:711-720.

25. Kim MJ, Kim JS, Seok JH. Effects of early-life stress on the structural and functional development of central nervous system: a review of the studies focusing on animal models. J Korean Neuropsychiatr Assoc 2016;55:75-88.

26. Millstein RA, Holmes A. Effects of repeated maternal separation on anxiety- and depression-related phenotypes in different mouse strains. Neurosci Biobehav Rev 2007;31:3-17.

27. Banqueri M, Méndez M, Arias JL. Behavioral effects in adolescence and early adulthood in two length models of maternal separation in male rats. Behav Brain Res 2017;324:77-86.

28. de Kloet ER, Sibug RM, Helmerhorst FM, Schmidt MV. Stress, genes and the mechanism of programming the brain for later life. Neurosci Biobehav Rev 2005;29:271-281.

29. Klein ZA, Romeo RD. Changes in hypothalamic-pituitaryadrenal stress responsiveness before and after puberty in rats. Horm Behav 2013;64:357-363.

30. Arakawa $\mathrm{H}$. Ethological approach to social isolation effects in behavioral studies of laboratory rodents. Behav Brain Res 2018;341:98-108.

31. Karelina K, DeVries AC. Modeling social influences on hu- 
man health. Psychosom Med 2011;73:67-74.

32. Cacioppo JT, Cacioppo S, Capitanio JP, Cole SW. The neuroendocrinology of social isolation. Annu Rev Psychol 2015; 66:733-767.

33. Campeau S, Davis M. Involvement of subcortical and cortical afferents to the lateral nucleus of the amygdala in fear conditioning measured with fear-potentiated startle in rats trained concurrently with auditory and visual conditioned stimuli. J Neurosci 1995; 15(3 Pt 2):2312-2327.

34. Bauer EP. Serotonin in fear conditioning processes. Behav Brain Res 2015;277:68-77.

35. Willner P, Gruca P, Lason M, Tota-Glowczyk K, Litwa E, Niemczyk M, et al. Validation of chronic mild stress in the Wistar-Kyoto rat as an animal model of treatment-resistant depression. Behav Pharmacol 2019;30(2 and 3-Spec Issue): 239-250.

36. Arp JM, Ter Horst JP, Loi M, den Blaauwen J, Bangert E, Fernández G, et al. Blocking glucocorticoid receptors at adolescent age prevents enhanced freezing between repeated cue-exposures after conditioned fear in adult mice raised under chronic early life stress. Neurobiol Learn Mem 2016;133: 30-38.

37. Nylander I, Roman E. Is the rodent maternal separation model a valid and effective model for studies on the early-life impact on ethanol consumption? Psychopharmacology (Berl) 2013; 229:555-569.

38. Czéh B, Fuchs E, Wiborg O, Simon M. Animal models of major depression and their clinical implications. Prog Neuropsychopharmacol Biol Psychiatry 2016;64:293-310.

39. Belovicova K, Bogi E, Csatlosova K, Dubovicky M. Animal tests for anxiety-like and depression-like behavior in rats. Interdiscip Toxicol 2017;10:40-43.

40. Pawlak CR, Karrenbauer BD, Schneider P, Ho YJ. The elevated plus-maze test: differential psychopharmacology of anxiety-related behavior. Emot Rev 2012;4:98-115.

41. Hu C, Luo Y, Wang $\mathrm{H}$, Kuang $\mathrm{S}$, Liang G, Yang $\mathrm{Y}$, et al. Re-evaluation of the interrelationships among the behavioral tests in rats exposed to chronic unpredictable mild stress. PLoS One 2017; 12:e0185129.

42. Bali A, Jaggi AS. Electric foot shock stress: a useful tool in neuropsychiatric studies. Rev Neurosci 2015;26:655-677.

43. Lee JH, Kimm S, Han JS, Choi JS. Chasing as a model of psychogenic stress: characterization of physiological and behavioral responses. Stress 2018;21:323-332.

44. Huot RL, Gonzalez ME, Ladd CO, Thrivikraman KV, Plotsky PM. Foster litters prevent hypothalamic-pituitary-adrenal axis sensitization mediated by neonatal maternal separation. Psychoneuroendocrinology 2004;29:279-289.

45. Own LS, Patel PD. Maternal behavior and offspring resiliency to maternal separation in C57Bl/6 mice. Horm Behav 2013; 63:411-417.

46. Weiss IC, Pryce CR, Jongen-Rêlo AL, Nanz-Bahr NI, Feldon J. Effect of social isolation on stress-related behavioural and neuroendocrine state in the rat. Behav Brain Res 2004;152: 279-295.

47. Flandreau EI, Toth M. Animal models of PTSD: a critical review. In: Vermetten E, Baker DG, Risbrough VB, editors. Behavioral neurobiology of PTSD. Cham:Springer International Publishing;2018. p.47-68.

48. Gallup Jr GG. Tonic immobility as a measure of fear in domestic fowl. Anim Behav 1979;27:316-317.

49. Northoff G. What catatonia can tell us about "top-down modulation": a neuropsychiatric hypothesis. Behav Brain Sci 2002;25:555-577.

50. Jelen P, Soltysik S, Zagrodzka J. 22-kHz ultrasonic vocalization in rats as an index of anxiety but not fear: behavioral and pharmacological modulation of affective state. Behav Brain Res 2003;141:63-72.

51. Budylin T, Guariglia SR, Duran LI, Behring BM, Shaikh Z, Neuwirth LS, et al. Ultrasonic vocalization sex differences in 5-HT1A-R deficient mouse pups: predictive phenotypes associated with later-life anxiety-like behaviors. Behav Brain Res 2019;373:112062.

52. Graham LK, Yoon T, Lee HJ, Kim JJ. Strain and sex differences in fear conditioning: $22 \mathrm{kHz}$ ultrasonic vocalizations and freezing in rats. Psychol Neurosci 2009;2:219-225.

53. Zhang L, Hu X, Li H, Li X, Yu T, Dohl J, et al. Updates in PTSD animal models characterization. In: Kobeissy $\mathrm{FH}$, editor. Psychiatric disorders: methods and protocols. New York, NY:Springer New York;2019. p.331-344.

54. Gallup Jr GG, Rager DR. Tonic immobility as a model of extreme states of behavioral inhibition. In: Sanberg PR, Ossenkopp $K P$, Kavaliers $M$, editors. Motor activity and movement disorders. Springer; 1996. p.57-80.

55. Marx BP, Forsyth JP, Gallup GG, Fusé T, Lexington JM. Tonic immobility as an evolved predator defense: implications for sexual assault survivors. Clin Psychol Sci Pract 2008; 15:74-90.

56. Kalaf J, Coutinho ESF, Vilete LMP, Luz MP, Berger W, Mendlowicz M, et al. Sexual trauma is more strongly associated with tonic immobility than other types of trauma - a population based study. J Affect Disord 2017;215:71-76.

57. Putnam FW. Dissociation in children and adolescents: a developmental perspective. New York, NY:Guilford Press; 1997.

58. Lloyd CS, Lanius RA, Brown MF, Neufeld RJ, Frewen PA, McKinnon MC. Assessing post-traumatic tonic immobility responses: the scale for tonic immobility occurring post-trauma. Chronic Stress 2019;3:247-292.

59. Volchan E, Rocha-Rego V, Bastos AF, Oliveira JM, Franklin C, Gleiser $\mathrm{S}$, et al. Immobility reactions under threat: a contribution to human defensive cascade and PTSD. Neurosci Biobehav Rev 2017;76(Pt A):29-38.

60. Yee N, Schwarting RK, Fuchs E, Wöhr M. Juvenile stress potentiates aversive 22-kHz ultrasonic vocalizations and freezing during auditory fear conditioning in adult male rats. Stress 2012;15:533-544.

61. Foilb AR, Lui P, Romeo RD. The transformation of hormonal 
stress responses throughout puberty and adolescence. J Endocrinol 2011;210:391-398.

62. de Kleine RA, Hagenaars MA, van Minnen A. Tonic immobility during re-experiencing the traumatic event in posttraumatic stress disorder. Psychiatry Res 2018;270:1105-1109.

63. Meaney MJ. Maternal care, gene expression, and the trans- mission of individual differences in stress reactivity across generations. Annu Rev Neurosci 2001;24:1161-1192.

64. Kuiling JME, Klaassen F, Hagenaars MA. The role of tonic immobility and control in the development of intrusive memories after experimental trauma. Memory 2019;27:772-779. 